\title{
The Near-Infrared Surface Brightness Distribution of NGC 4696
}

\author{
Pablo Arnalte Mur ${ }^{\mathrm{A}, \mathrm{B}, \mathrm{C}}$, Simon C. Ellis ${ }^{\mathrm{A}}$, and Matthew Colless ${ }^{\mathrm{A}}$ \\ A Anglo-Australian Observatory, Epping NSW 2121, Australia \\ ${ }^{B}$ Facultat de Física, Universitat de València, 46100 Burjassot, València, Spain \\ C Corresponding author. Email: parmur@alumni.uv.es
}

Received 2005 November 8, accepted 2006 January 16

\begin{abstract}
We present $H$-band observations of the elliptical galaxy NGC 4696, the brightest member of the Centaurus cluster of galaxies. We have measured its light profile, using a two-dimensional fitting algorithm, out to a radius of $180 \operatorname{arcsec}\left(37 h_{70}^{-1} \mathrm{kpc}\right)$. The profile is well described by a de Vaucouleurs law, with an effective radius of $35.3 \pm 1.0 h_{70}^{-1} \mathrm{kpc}$. There is no need for the extra free parameter allowed by a Sérsic law. Allowing for a variation of $0.3 \%$ in the sky level, the profile obtained is compatible with data from the TwoMicron All Sky Survey (2MASS). The profile shows no sign of either a truncation or an extended halo.
\end{abstract}

Keywords: galaxies: elliptical and lenticular, $\mathrm{cD}$ - galaxies: fundamental parameters — galaxies: halos galaxies: individual (NGC 4696) — galaxies: photometry — galaxies: structure

\section{Introduction}

Surface brightness profiles of elliptical galaxies are normally well described by either a de Vaucouleurs law (de Vaucouleurs 1948) or a Sérsic law (Sérsic 1968). However, the situation can be more complicated when we consider a galaxy embedded in a cluster. In this case, the outer parts of the profile may be modified due to tidal interactions with neighboring galaxies, giving a 'truncated' profile. A different case is often encountered in elliptical galaxies that are the brightest cluster member (BCM) and are located at the cluster centre, many of which have an extended envelope or halo (Schombert 1987; Gonzalez, Zabludoff, \& Zaritsky 2005). These galaxies are known as 'central dominant' (cD) galaxies. The extended envelope is observed as a deviation of the profile from a de Vaucouleurs or Sérsic law at large radii, and appears to correspond to light from stars orbiting in the cluster potential and not bound to the central galaxy (Bahcall 1977; Gonzalez et al. 2005). Simulations indicate that the stars in the envelope may, on average, be older than the stars in the galaxy (Murante et al. 2004).

NGC 4696 is the BCM of the Centaurus cluster. It is an elliptical galaxy located near the centre of the cluster. It is sometimes described as a $\mathrm{cD}$ galaxy, although no evidence has been found up to now of an extended halo.

Previous measurements of the light profile of NGC 4696 at optical wavelengths include those of Schombert (1987) and Jerjen \& Dressler (1997), both obtained from photographic plates. Jerjen \& Dressler (1997) measured the light profile out to a radius of approximately $80 \mathrm{arcsec}$ ( $\approx 16 h_{70}^{-1} \mathrm{kpc}$ ) whereas the profile obtained by Schombert (1987) reaches much further, out to a radius of about $9 \operatorname{arcmin}\left(\approx 110 h_{70}^{-1} \mathrm{kpc}\right)$. Neither observation showed signs of an extended envelope. The profile from Schombert (1987) followed an $r^{1 / 4}$ law (de Vaucouleurs profile) out to a radius of approximately $5 \mathrm{arcmin}$, and a truncation or 'distention' of the profile was found for larger radii.

Measurements of the near-infrared (NIR) profile were obtained by the Two-Micron All Sky Survey (source 2MASX J12484927-4118399 in the all-sky extended source catalog $\left.{ }^{1}\right)$. Light profiles were obtained in three bands $(J, H$, and $K)$ for radii up to approximately $3 \operatorname{arcmin}$. This corresponds to surface brightnesses $\mu_{H} \leq 24$ mag $\operatorname{arcsec}^{-2}$ in the $H$-band.

The intra-cluster medium (ICM) of the Centaurus cluster has been studied using X-ray emission by the ROSAT and ASCA satellites. Allen \& Fabian (1994), using data from ROSAT, found a correlation between the structure ellipticity and centroid - of the X-ray emission and optical observations (Sparks, Macchetto, \& Golombek 1989) of the central galaxy NGC 4696. Ikebe et al. (1999) used data from both satellites, and described the X-ray emitting gas in the central region $(r \leq 3 \mathrm{arcmin})$ according to a two-phase model. They identified the cool phase with the inter-stellar medium (ISM) of NGC 4696, and the hot phase with the ICM of the Centaurus cluster. These relationships between the central galaxy and the ICM could be an indication of the presence of an extended halo.

The previous optical and NIR work pose a quandary in that the form of the profiles measured range from de Vaucouleurs to exponential. Thus the aim of the present work was to observe the light profile of NGC 4696 in the NIR out to large radii, to study the functional form of the profile (de Vaucouleurs or Sérsic law), to measure the parameters of the profile, and to seek evidence for either a truncation due to tidal interactions or an extended envelope following the cluster potential. The main advantage of the

\footnotetext{
${ }^{1}$ www.ipac.caltech.edu/2mass/
} 
NIR over optical studies is that the light is dominated by old stars, and thus the NIR profile will better trace the bulk of the stellar mass of the galaxy. Furthermore, the extinction due to dust is smaller in the NIR than at optical wavelengths.

Another motivation for this work is the application to studying radial color distributions, through coupling with optical data, to understand the distribution of stellar populations throughout BCMs. Through comparisons with simulations (e.g. Sommer-Larsen, Romeo, \& Portinari 2005), such studies would help to constrain BCM and cluster formation theories and the enrichment of the intracluster medium (e.g. Lin \& Mohr 2004).

We describe our observational data in Section 2 and explain reductions and calibrations in Section 3 and the method used to fit the profile models in Section 4. Section 5 presents the results and Section 6 summarizes our conclusions.

Throughout the paper we use a Hubble constant of $H_{0}=70 h_{70} \mathrm{~km} \mathrm{~s}^{-1} \mathrm{Mpc}^{-1}$. We have used a redshift of $z=0.00987 \pm 0.00005$ (de Vaucouleurs et al. 1991, which assumes no peculiar velocity) which equates to a distance of $42.3 \pm 0.2 h_{70}^{-1} \mathrm{Mpc}$; the angular scale at this distance is $0.205 \pm 0.001 \mathrm{kpc} \operatorname{arcsec}^{-1}$. Distances derived from surface brightness fluctuations, taking into account the peculiar motions of the Cen 30 and Cen 45 components of the Centaurus cluster and the Hydra cluster, gave a distance of $42.5 \pm 3.2 \mathrm{Mpc}$, consistent with the value used here (Mieske, Hilker, \& Infante 2005).

\section{Observations}

Observations were made on 2005 February 16, using the AAO Infrared Imager \& Spectrograph (IRIS2) at the 3.9-m Anglo-Australian Telescope (AAT). Conditions during the night were photometric, but the seeing was 2.5 arcsec. Data were obtained using the $H$-filter. The detector in IRIS2 is a $1024 \times 1024$ Rockwell HAWAII- 1 $\mathrm{HgCdTe}$ infrared detector. The detector scale is 0.4486 arcsec pixel ${ }^{-1}$, giving a field of view of $7.7 \times 7.7 \mathrm{arcmin}^{2}$ (Tinney et al. 2004).

We obtained a total of 99 frames of the field around NGC 4696 using a nine-point dither. Each of these frames is an average of six exposures of $10 \mathrm{~s}$ each. To measure the light profile to the full extent allowed by the detector, NGC 4696 was placed in the south-east corner of the field of view. We also obtained observations of three photometric standard stars (UKIRT Faint Standards FS129, FS132, and FS135; Hawarden et al. 2001), taking five 10-s exposures in each case. In order to model the point spread function (PSF), a bright star at a similar airmass to the science field was observed over 93 2.5-s exposures. As explained in Section 3 , extra data was needed for the flat-fielding process. This was obtained from other $H$-band observations taken on 2005 February 11 during the same run.

\section{Reduction and Calibration}

The data reduction was carried out using the IRAF (iraf.noao.edu) and ORAC-DR (www.oracdr.org) software.
The steps followed were subtraction of dark current, flat-fielding, registering to obtain the correct astrometric offsets, and combination of the 99 frames.

In the first step, the dark current in each pixel was subtracted from each of the raw frames. This value was obtained from a dark frame taken using the same exposure time (10 s) as the science frames. The typical value of the dark current was about 3 ADU (about 15 electrons).

To take into account the difference in response between pixels in the detector, it was necessary to flat-field the frames. It was not possible to obtain a flat field directly from our science field, as the large extent of NGC 4696 meant that any flat field obtained from the science field images would retain some contribution from the galaxy.

The flat field used was therefore derived from $\mathrm{H}$ band observations made on February 11, during the same IRIS2 observing run. These were used instead of other observations from February 16 for a variety of reasons. In the case of the standard photometric stars, other very bright stars in the field caused artifacts in the flat field. In the case of the 'PSF star', the accuracy of the resulting flat field was limited by the short exposure time. Using all the 10-s exposed frames from February 11 that did not contain bright stars, we could construct a flat field with a total exposure time of about $58 \mathrm{~min}$.

The flat field from these frames was obtained using ORAC-DR. For each of the fields, the frames were mediancombined to obtain a first estimate for the flat. Then the original frames were divided by this estimate, objects were detected and masked, and the frames were combined again to obtain the final flat for the field. The flat used was an average of these flats. The dark-subtracted frames were divided by this flat before being registered and combined.

Before combining the 99 frames, registration was carried out to find the true offsets between the frames. This step was done using the XREGISTER task in IRAF, which performs a cross-correlation between objects in the field. Knowing these offsets, the IRAF task COMBINE was used to sigma-clip and median-combine the frames, obtaining the final image. The base sky-level is not subtracted, but rather an additive zero point is applied to each frame to account for differences in sky level. This zero point was calculated as the mode of the same physical region of sky in all the frames. A 'sigma image' containing the standard deviation of the values corresponding to each pixel in the final image was also derived.

The final image obtained using this reduction procedure is shown in Figure 1. Large-scale (of the order of arcmin) variations in the resulting background that do not correspond to the galaxy's light were observed at the level of $0.4 \%$ of the mean sky background, corresponding to an uncertainty of approximately $0.004 \mathrm{mag} \operatorname{arcsec}^{-2}$. These variations impose the effective limit on the radius to which we were able to accurately measure the surface brightness profile.

The photometric zero point for our observations was obtained from the photometric standards we observed. Our instrumental magnitudes for these stars were compared to 


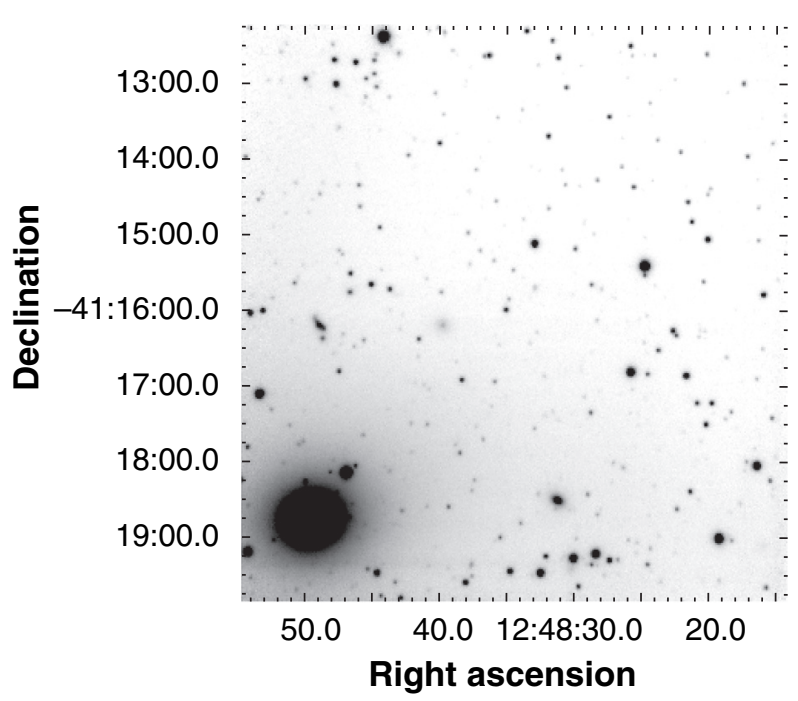

Figure 1. Final image used for the analysis. North is to the top and east is to the left. The field of view is $7.7 \times 7.7 \mathrm{arcmin}^{2}$. NGC 4696 is located near the south-east (bottom left) corner.

their $H$-magnitude in the 2MASS catalog. The zero point obtained has an associated error of \pm 0.03 mag.

\section{Data Analysis}

Two further elements were needed prior to fitting the galaxy's profile - an estimate of the PSF in the observations, and a mask for the other objects in the field. The profile could then be studied using the two-dimensional fitting algorithm GALFIT (Peng et al. 2002).

The PSF was obtained from the profile of a bright star observed immediately after NGC 4696 at a similar airmass to the science field, and hence with comparable seeing. A region of the image centred on the PSF star and covering $150 \times 150$ pixels $(\approx 67 \times 67 \operatorname{arcsec})$ was used directly to model the PSF. This PSF was convolved with the different galaxy profile models before comparing them with the data during the fitting process.

In order to test the robustness of the fitting process to the exact PSF, the fits were repeated with artificial PSF images with varying FWHM, and Moffat and Gaussian profiles. Large differences were found between a Moffat and Gaussian profile, but the FWHM had only a small effect $(\approx 2.5 \%)$ on the fitted value of the effective radius $r_{\mathrm{e}}$. Since we used an image of a star for the PSF, the shape of the profile will be accurately determined, and thus our fits should be robust to any changes in the PSF FHWM due to any differences in observing conditions. Furthermore, the PSF will only strongly affect the core region of the galaxy profile, which was not used in the final fit.

A mask was needed to exclude other objects in the field affecting the profile fit. For the most of the field, objects were detected using SEXTRACTOR (Bertin \& Arnouts 1996). However, objects close to the core of NGC 4696 were not correctly detected, so objects in a region out to about 90 arcsec from the centre of the galaxy were detected and masked by eye.

The galaxy surface brightness distribution was fitted using GALFIT. The models considered were a de Vaucouleurs profile and the more general Sérsic profile. Different initial parameters were used to test the stability of the fitting algorithm. The 'sigma' image obtained from the combining process was used as the input error assigned to each pixel.

There is a strong dependence of the fit results on the correct determination of the sky level. We therefore kept the sky level as an additional free parameter in all the fits. In order to test the dependence on the sky level used, we also fitted profiles with the sky level held constant at $\pm 0.2 \%$ of the best fitting value to reflect the $0.4 \%$ variation seen across the image. The results are given in Table 1.

The geometric parameters of the galaxy (axis ratio and orientation of the semi-major axis) were obtained from the initial fits. The axis ratio is $b / a=0.83 \pm 0.01$ and the position angle is $\theta=100^{\circ} \pm 2^{\circ}$ (measured north through east). These values were kept constant in the following fits and were used to construct masks limiting the region to fit, setting both the minimum and maximum radius for each fit.

Masking the core of the galaxy was essential, as some complex structure was observed in that region, which is unrelated to the rest of the galaxy's profile (Crawford et al. 2005). The region masked was an ellipse with axis ratio and orientation as above and semi-major axis of 18 arcsec.

The goodness-of-fit can be evaluated from the $\chi^{2}$ values of the fit and the number of degrees of freedom $(v)$ in the model. The value of $\chi^{2}$ is calculated directly by GALFIT. However, due to the correlation between neighboring pixels caused by the PSF, $v$ cannot be calculated

Table 1. Surface brightness profile parameters

\begin{tabular}{|c|c|c|c|}
\hline Parameter & de Vaucouleurs & Sérsic & Systematic errors $^{\mathrm{A}}$ \\
\hline $\mathrm{m}[\mathrm{mag}]$ & $6.25 \pm 0.06$ & $6.13 \pm 0.10$ & $(4.86,6.94)$ \\
\hline$\mu_{0}\left[\mathrm{mag} \operatorname{arcsec}^{-2}\right]$ & $12.29 \pm 0.07$ & $11.8 \pm 0.5$ & $(8.37,14.51)$ \\
\hline$\mu_{\mathrm{e}}\left[\mathrm{mag} \operatorname{arcsec}^{-2}\right]$ & $20.62 \pm 0.07$ & $20.90 \pm 0.22$ & $(23.75,19.36)$ \\
\hline$n$ [Sérsic index] & 4 & $4.3 \pm 0.3$ & $(7.3,2.4)$ \\
\hline$r_{\mathrm{e}}[\operatorname{arcsec}]$ & $172 \pm 5$ & $204 \pm 20$ & $(1199,76)$ \\
\hline$r_{\mathrm{e}}\left[h_{70}^{-1} \mathrm{kpc}\right]$ & $35.3 \pm 1.0$ & $42 \pm 4$ & $(246,16)$ \\
\hline$\chi_{v}^{2}$ & 1.170 & 1.167 & \\
\hline
\end{tabular}

${ }^{\mathrm{A}}$ Limits based on fitting a Sérsic profile with sky values fixed at $\pm 0.2 \%$ the best fitting value. 
directly as the number of pixels minus the number of parameters. To overcome this, artificial images were created from models with the same parameters as the fits obtained and noise added at the same level as our data. These images were then smoothed using a PSF of the same FWHM as observed. Fits obtained from the resulting smoothed images give an estimate of the effect of the correlation. Values of the reduced $\chi^{2}\left(\chi_{v}^{2}=\chi^{2} / v\right)$ quoted below take this correction into account.

\section{Results and Discussion}

Successive fits were performed in regions limited by an ellipse with the same geometric parameters as the galaxy and a varying semi-major axis. The maximum distance at which the fit was reliable was estimated using three indicators: the values of $\chi_{v}^{2}$, visual inspection of the model-subtracted image, and the variation of flux with angle in successive elliptical rings. The maximum allowed value for the semi-major axis distance was 180 arcsec ( $\left.37 h_{70}^{-1} \mathrm{kpc}\right)$. All the results below refer only to fits within this region.

The total magnitude $(m)$, central surface brightness $\left(\mu_{0}\right)$, effective radius $\left(r_{\mathrm{e}}\right)$, and surface brightness at $r_{\mathrm{e}}\left(\mu_{\mathrm{e}}\right)$ obtained for de Vaucouleurs and Sérsic profiles fitted to the studied region are shown in Table 1 . The extra free parameter in the Sérsic model (the index $n$ ) scarcely improves the value of $\chi^{2}$, and the index obtained is very close to the de Vaucouleurs value of $n=4$. We therefore conclude that this extra free parameter is not needed and that the light profile of NGC 4696 is well described (in the region within 180 arcsec) by a de Vaucouleurs law. A plot of the observed surface brightness profile of NGC 4696, together with this best fit, is shown in Figure 2.

The fit was repeated introducing variations of the geometric parameters as well as the masking of the core region in order to test the robustness of the results. The main differences were found when varying the core masking, probably due to the presence of some extra structure close to the centre of the galaxy or uncertainties in the PSF. Errors quoted in Table 1 correspond to these variations. The differences found when varying the geometric parameters (orientation and axis ratio) were smaller than these errors.

The profile obtained was compared to that obtained in the $H$-band from $2 \mathrm{MASS}$. The comparison is shown in Figure 3. There is a clear difference in the slopes of the profiles beyond the galaxy core. 2MASS fit a Sérsic profile with an index of $n=1.11$, whereas we find a profile that is well fit by an $n=4$ de Vaucouleurs model. The difference between our result and the 2MASS result is likely to be a consequence of the sky background estimation. It was found that the slope of the outer profile was very sensitive to the precise sky level used. If we subtract a sky level higher by only $\approx 0.3 \%$ than that obtained from the best fit, the difference was essentially eliminated. Note that this difference could be explained by the large scale variations of $\approx 0.4 \%$ observed in our final image, whereas $2 \mathrm{MASS}$ has more optimally observed flat-fields. However, our deeper observations

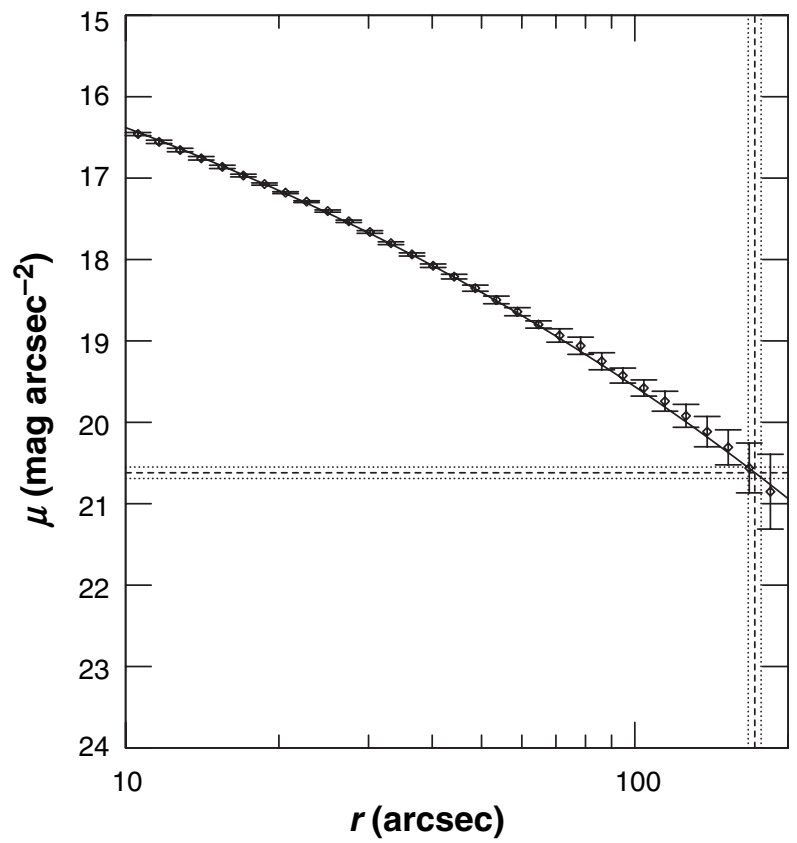

Figure 2 Comparison between the observed profile (points) and the fitted de Vaucouleurs model (solid line). Each point in the plot corresponds to the average surface brightness of the pixels within an elliptical annulus (obtained with IRAF task ELLIPSE); $r$ is the semimajor axis distance to that annulus. The error bars correspond to the dispersion in surface brightness of these pixels. The dashed (dotted) lines correspond to the values of $r_{e}\left(\mu_{e}\right)$ and their uncertainties.

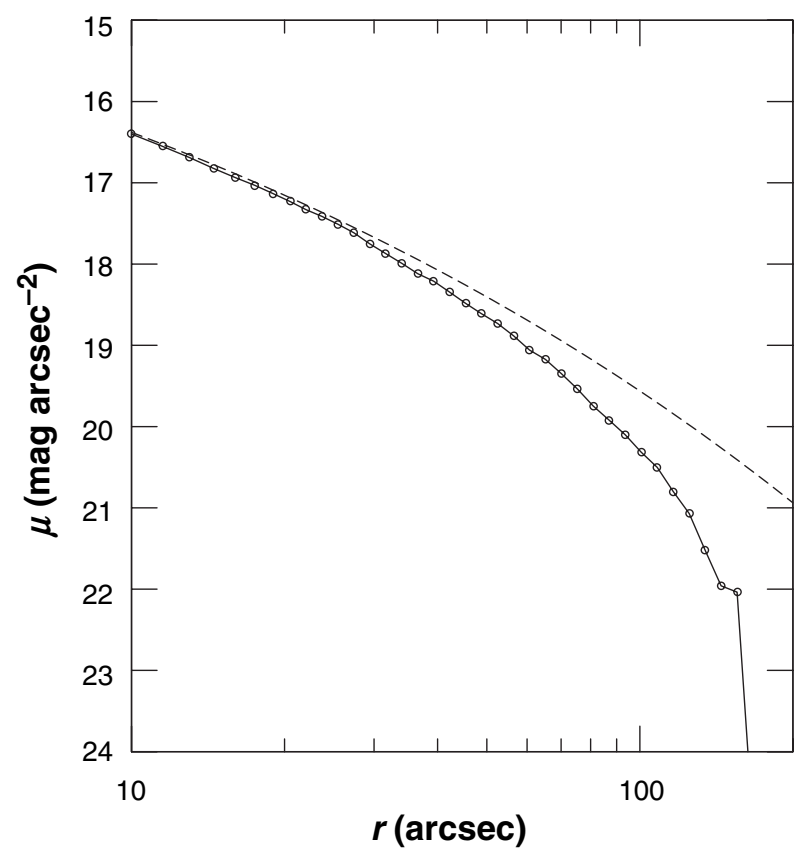

Figure 3 Comparison with 2MASS surface brightness profile. The points and solid line are the 2MASS profile and the dashed line is our fitted de Vaucouleurs profile (with parameters as in Table 1).

are in better agreement with previous work done in the optical; Schombert (1987) finds a good fit with a de Vaucouleurs model to $r \approx 5$ arcmin, and Jerjen \& Dressler (1997) fit a Sérsic profile with $n=2.04$. 
The extremely sensitive nature of the fits to the sky level (see Table 1) means that the previous uncertainty in the profile of NGC 4696 is still unresolved. Future observations could improve on the present work through either off-source blank field observations interleaved with the galaxy observations, or drift scan observations, as in 2MASS but deeper, to better estimate the flat-field and reduce the uncertainty in the sky levels.

\section{Conclusions}

The NIR light profile of NGC 4696 in the $H$-band was measured out to a distance of $180 \operatorname{arcsec}\left(37 h_{70}^{-1} \mathrm{kpc}\right)$ along the semi-major axis. This limit was imposed by systematic errors in the flat-fielding process. The obtained profile is well described by a de Vaucouleurs law, with no signs of either truncation or an extended halo. These observations agree, up to an uncertainty of $0.3 \%$ in the sky level, with the $H$-band profile obtained from 2MASS.

\section{Acknowledgments}

This paper is based on data obtained with the AngloAustralian Telescope. It also makes use of data products from 2MASS, which is a joint project of the University of Massachusetts and the Infrared Processing and Analysis Center, California Institute of Technology, funded by the National Aeronautics and Space Administration and the National Science Foundation.

\section{References}

Allen, S. W., \& Fabian, A. C. 1994, MNRAS, 269,409

Bahcall, N. A. 1977, ARA\&A, 15, 505

Bertin, E., \& Arnouts, W. 1996, A\&AS, 117, 393

Crawford, C. S., Hatch, N. A., Fabian, A. C., \& Sanders, J. S. 2005, MNRAS, 363, 216

de Vaucouleurs, G. 1948, AnAp, 11, 247

de Vaucouleurs, G., de Vaucouleurs, A., Corwin Jr., H. G., Buta, R. J., Paturel, G., \& Fouque, P. 1991, Third Reference Catalogue of Bright Galaxies, Vol 3.9 (New York: Springer)

Gonzalez, A. H., Zabludoff, A. I., \& Zaritsky, D. 2005, ApJ, 618, 195

Hawarden, T. G., Leggett, S. K., Letawsky, M. B., Ballantyne, D. R., \& Casali, M. M. 2001, MNRAS, 325, 563

Ikebe, Y., Makishima, K., Fukazawa, Y., Tamura, T., Xu, H., Ohashi, T., \& Matsushita, K. 1999, ApJ, 525, 58

Jerjen, H., \& Dressler, A. 1997, A\&AS, 124, 1

Lin, Y.-T., \& Mohr, J. J. 2004, ApJ, 617, 879

Mieske, S., Hilker, M., \& Infante, L. 2005, A\&A, 438, 103

Murante, G., et al. 2004, ApJ, 607, L83

Peng, C. Y., Ho, L. C., Impey, C. D., \& Rix, H.-W. 2002, AJ, 124, 266

Schombert, J. M. 1987, ApJS, 64, 643

Sérsic, J. L. 1968, Atlas de galaxias australes (Córdoba: Observatorio Astronómico)

Sommer-Larsen, J., Romeo, A. D., \& Portinari, L. 2005, MNRAS, 357,478

Sparks, W. B., Macchetto, F., \& Golombek, D. 1989, ApJ, 345, 153

Tinney, C. G., et al. 2004, in Ground-Based Instrumentation for Astronomy (Eds Moorwood, A. F. M., \& Masamori, I.), p. 998 (Bellingham: SPIE) 